\title{
Solar Tracker Based Power Optimization In Performance Of Robotic Vehicle
}

\author{
M.Kanimozhi ${ }^{1}$,, M.Balabharathi ${ }^{2}$., K.S.Tamilselvan ${ }^{3}$ \\ ${ }^{1} P G$ Scholar., Dept. of Electronics and Communication Engineering, Velalar College of Engineering and \\ Technology., ${ }^{2} P$ GSCholar., Dept. of Electronics and Communication Engineering, Velalar College of \\ Engineering and Technology., ${ }^{3}$ Asst.Professor., Dept. of Electronics and Communication Engineering., Velalar \\ College of Engineering and Technology.
}

Abstract: Main motivation of this project is to design a wireless based solar powered robotic system in order to optimize the power by improving the utilization of solar power. A new idea has been presented for wireless solar powered mobile robotic system which consists of two parts, first is the solar powered mechanical robot and the second is zigbee wireless based control section which controls and moves the first part. By this system various mobility control has been performed and proved the ability to control the group of robots by controlling the group of activities to be done in the availability of solar power.

Keywords -Solar Tracker, Robotic System, Light Dependent Resistor, Stepper motor.

\section{Introduction}

In earlier days, batteries were used to provide power to the robotic system. But, the batteries when depleted could not be recharged, thus affecting the performance of the autonomous robotic system. To overcome this, fuel cell based power supply system was introduced, which replaced the batteries in portable applications. The complexity increased because of large sized fuel cells.

The, solar powered robotic systems are often used for many years. However, when there is scarcity of sunlight the batteries in line could not be recharged when depleted. So, rechargeable batteries came into account for the first time in the mars exploration rover. Nevertheless, the need for greater operation autonomy by spirit and opportunity was solved by means of larger deploy of solar cells.

Solar power is the conversion of sunlight into electricity. Photovoltaics are used for theconversion of sunlight into electricity either directly or indirectly with concentrated solar power(CSP), which normally concentrates the sun's energy to boil water which is then used to generate power. Photovoltaics were initially used to power small and medium sized applications, that are powered by a single solar cell to off-grid homes powered by a photovoltaic array.

Today, the declining cost and increasing efficiency of solar energy technology has given rise to practical applications on earth from powering personal devices to provide utility-scale power. Solar energy provides a huge advantage for satellites because the addition of fuel supply for satellites can be avoided while launching them into orbit.

But the advantages on earth are even greater: solar-generated energy provides abundant and pollutionfree energy that's not dependent on fuel-delivery antecedent, foreign relations or the price machinations of energy brokers and large firm. Moreover, solar power generation provides energy when and where we need it, and is highly scalable to match the electrical demand. Since solar cells are reliable and easy to maintain.

\section{Existing Methods}

Alexander N. Wilhelm, et.al [1], proposed fuel cell based power system for mobile robot. The fuel system includes a fuel stack which converts fuel into electric power. It also includes fuel for the fuel cell stack and a controller which controls the operation of the fuel cell system. The fuel system acts as an interface to the portable computing device and a bidirectional communication between the portable computing device and the controller for the fuel cell system. The fuel based power supply system increases the run time with simple refueling. Thus the need for lengthy recharge is avoided. The power for the robotic system remains available as long as fuel is available.

But, the fuel cells were large and extremely expensive to manufacture. But, just like these products the cost of fuel cells will quickly come down to consumer-affordable levels with mass production. We are currently in a evolution period now, where many fuel cell companies are investigating literally hundreds of millions of dollars to gear up for mass manufacturing at the same time they are trying to develop a variety of markets for their products. 
Currently there is no hydrogen infrastructure to supply coast-to-coast delivery of hydrogen fuels. Technologies are being developed for alternative fuel storage and delivery methods. But those technologies can lower the overall efficiency of the fuel cell by $1 / 4$ and release a small amount of pollutants.

Iain A. Anderson, et.al [2], proposed artificial muscles based on the dielectric elastomer actuator (DEA) for autonomous robotic systems. Dielectric elastomer (DE) actuators are popularly referred to as artificial muscles because their impressive actuation effort and speed, low density, flexible nature, and silent operation capture many of the desirable physical properties of muscle. Unlike standard robots and machines, whose contrivance and drive systems rapidly become very complex as the number of degrees of freedom increases, groups of Dielectric Elastomer artificial muscles have the potential to generate rich motions combining many translational and rotational degrees of freedom.

The Dielectric Elastomer Generator (DEG) with Self Priming Circuit(SPC) can able to convert even a small microbial organism into electricity. In addition, they have the ability to produce electricity from displacement. But departing from the high stiffness algorithm of electromagnetic motors and gearboxes leads to new control challenges, and for soft machines to be truly nimble like their biological analogues, they need precise control. For an autonomous robot such as EcoBot, a good supply of electrical energy at high voltage would enable the more general use of DEA for on-board systems and there are a number of applications that DEA could be applied to. The main drawback of this system is that the time and complexity increases.

Torabi.H.B, et.al [3], A Solar Power System for experimental unmanned aerial vehicle (UAV) is designed and summarized. A microcontroller based controller was developed to collect the output power data of power source to be send to the ground station. solar cells should not be bended,so the surface should be smooth enough for installing solar cells on the surface of the wing,an airfoil. This airfoil should also be a low-Reynoldsnumber airfoil.

J. E. Gonz' alez Ramos [4], Battery charging optimization with steerable solar cells has been proposed to estimate the battery lifetime and power cost of shallow water networks in terms of four independent parameters (1) intern-ode distance; (2) transmission frequency; (3) frequency of data updates; and (4) number of nodes per cluster. A steerable solar panel array would track the sun as it moved across the sky, but we are not aware of any economically available domestic units in this country. Steerable arrays are costly. They need to be strong enough to resist high winds and require an adequate foundation.

David Appleyard, et.al [5], Solar energy also has the plausible to be the major energy supply in the forth coming. Solar tracker is an automated solar panel that actually follows the Sun to increase the power.

A. B. Afarulrazi, et.al [6], automatic Solar Tracker Robot (STR) which is capable to track maximum light intensity was designed and developed. The efficiency of the solar energy conversion can be optimized by receiving maximum light on the solar panel. The servo motor that is used to maintain the panels position in accordance with the suns position is quite complex and provides faster rotation. T. Jinayim, et.al [7], proposed fixed solar cells module to energize White LED light sources that are operated by directly connected White LED with current limiting resistors, which increases the power consumption.

Adrian Catarius [8], proposed azimuth-altitude dual axis solar tracker to provide an efficient solar distributed generated system. The dual axis solar tracker increases complexity of the system.Tom'as de J. Mateo Sanguino,et.al [9], presents the construction of a solar tracking mechanism aimed at increasing the rover's power regardless of its mobility and it proposes an alternative design of power system performance based on a pack of two batteries.

\section{Proposed Method}

The proposed system uses Master and Slave based Microcontrollers. The Master controller PIC16F877A senses the light intensity from two different directions using LDR (Light Dependent Resistors). The panel's direction is adjusted according to the sunlight direction using a stepper motor. The exploration robot has two-fingered arm to pic and place the objects. The robot is controlled using Zigbee protocol. The Battery status will be continuously measured and sent back to the monitoring unit through Zigbee. The monitoring section consist a PC with Zigbee transceiver, VB based user interface used to control the unmanned exploration vehicle. Since we are using solar-based system, power consumption is reduced. 


\section{BLOCK DIAGRAM:}

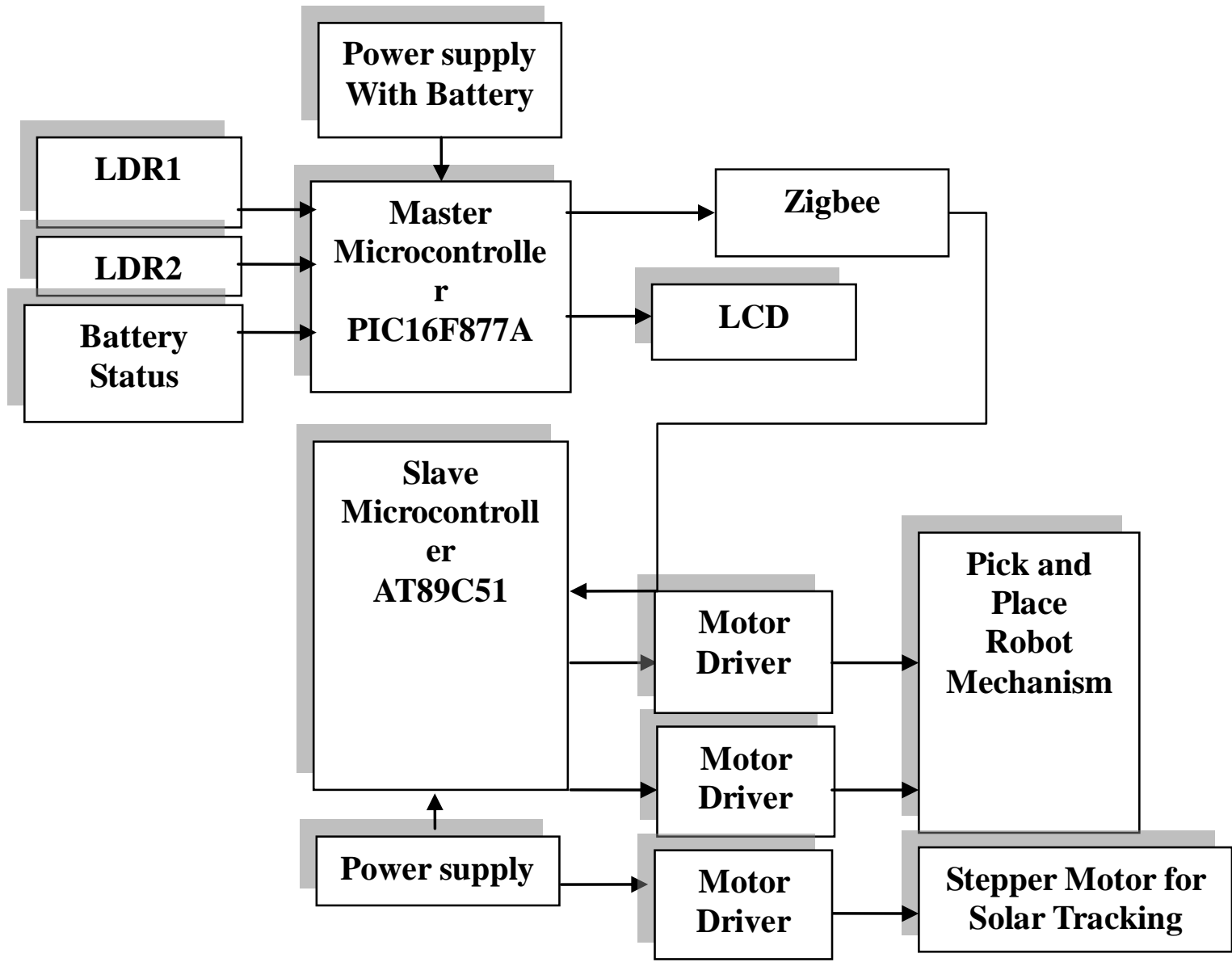

CONTROL AND MONITORING SECTION:

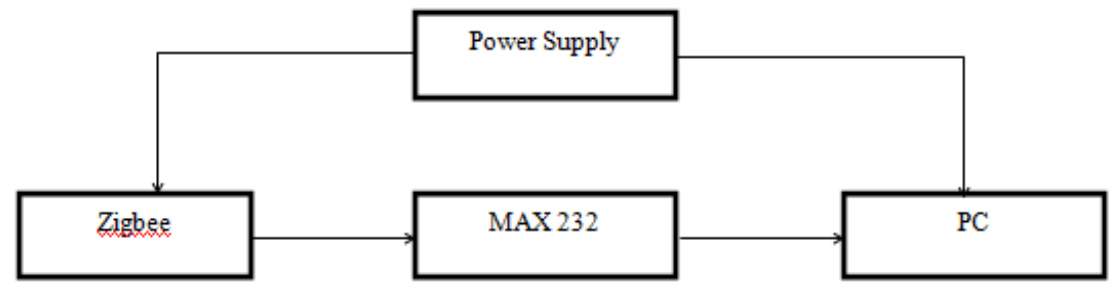

\section{CONTROL UNITS:}

In the control section, we are going to view the operation on the monitor regarding the robot movements.

\section{Power Supply Unit}

The supply of $5 \mathrm{~V}$ DC is given to the system which is converted from $230 \mathrm{~V}$ AC supply. Firstly, the step down transformer will be used here for converting the $230 \mathrm{~V} \mathrm{AC}$ into $12 \mathrm{~V} \mathrm{AC}$. The microcontroller will support only the DC supply, so the AC supply will be converted into DC using the bridge rectifier. The output of the rectifier will have ripples so we are using the 2200uf capacitor for filtering those ripples. The output from the filter is given to the 7805 voltage regulator which will convert the $12 \mathrm{~V} \mathrm{DC}$ into $5 \mathrm{~V} \mathrm{DC}$. The output from the regulator will be filtered using the 1000 uf capacitor, so the pure $5 \mathrm{~V}$ DC is getting as the output from the power supply unit. Here we are using the PIC microcontroller which will be capable of getting the supply of 5V DC so we have to convert the $230 \mathrm{~V}$ AC supply into $5 \mathrm{~V}$ DC supply.

\section{Microcontroller Unit}

PIC16F877A Microcontroller

The PIC16F877A CMOS FLASH-based 8-bit microcontroller is upward compatible with the PIC16C5x, PIC12Cxxx and PIC16C7x devices. It features 200 ns instruction execution, 256 bytes of EEPROM 
data memory, self programming, an ICD, 2 Comparators, 8 channels of 10-bit Analog-to-Digital(A/D) converter, 2capture/compare/PWM functions, a synchronous serial port that can be configured as either 3-wire SPI or 2-wire I2C bus, a USART, and a Parallel Slave Port.

\begin{tabular}{llll}
\hline Standard & $\begin{array}{l}\text { Zigbee } \\
\mathbf{8 0 2 . 1 5 . 4}\end{array}$ & $\begin{array}{l}\text { Wi-Fi } \\
\mathbf{8 0 2 . 1 1 b}\end{array}$ & $\begin{array}{l}\text { Bluetooth } \\
\mathbf{8 0 2 . 1 5 . 1}\end{array}$ \\
\hline Transmission Range (meters) & $1-100 *$ & $1-100$ & $1-10$ \\
\hline Battery Life (days) & $100-1,000$ & $0.5-5.0$ & $1-7$ \\
\hline Network Size (\# of nodes) & $>64,000$ & 32 & 7 \\
\hline Application & Monitoring \& Control & Web, Email, Video & Cable Replacement \\
\hline Stack Size (KB) & $4-32$ & 1,000 & 250 \\
\hline Throughput kb/s) & $20-250$ & 11,000 & 720
\end{tabular}

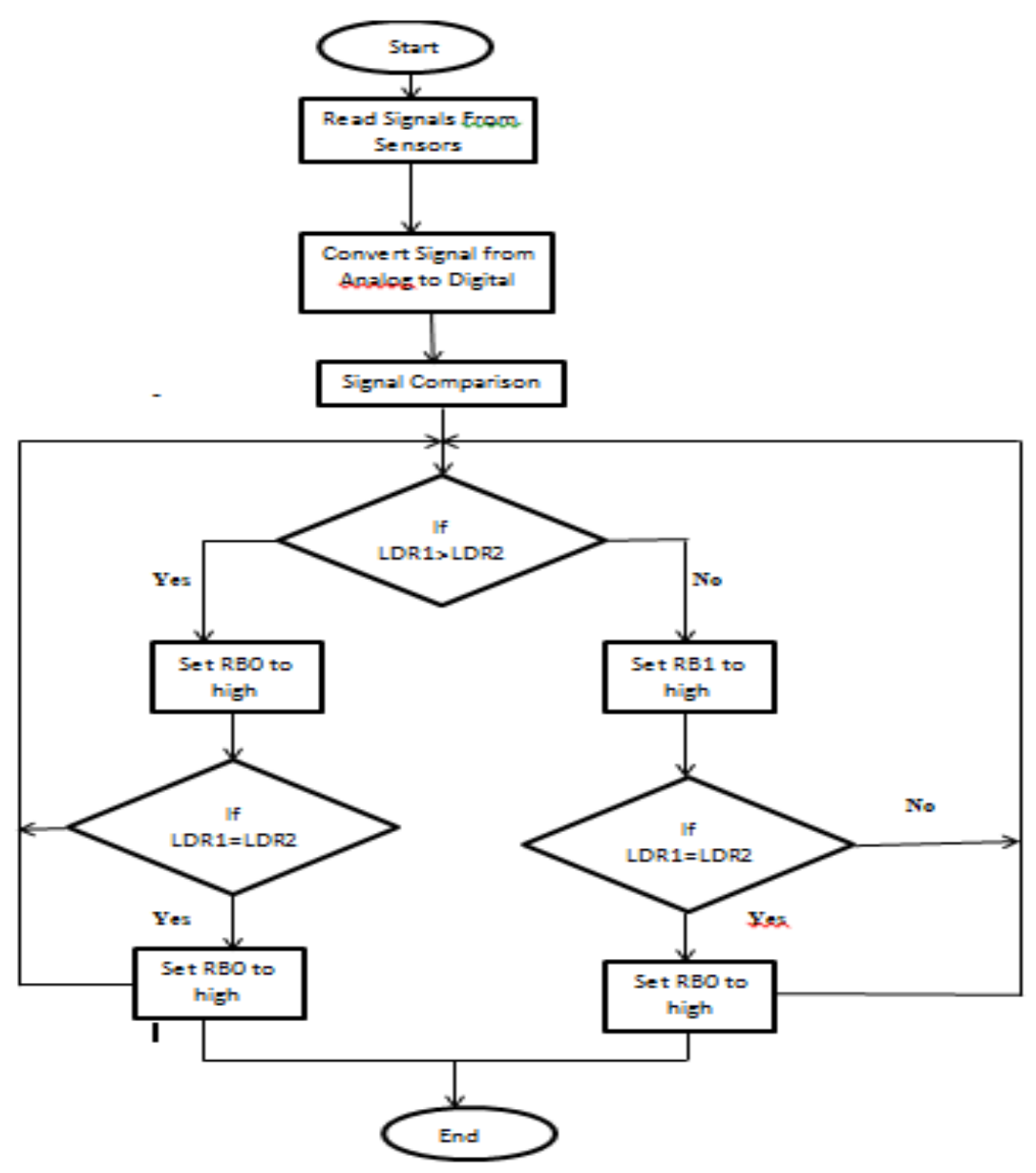

\section{AT89C51 Microcontroller}

The microcontroller used in our project is the AT89C51 microcontroller which is used to control the motors in our robot. Depending upon the Zigbee communication the commands received from the user PC we are going to the decide in which the robot has to move. The whole programming will be written in the microcontroller itself depending upon our requirement. This microcontroller is the cheap and best controller for controlling these types of operations.

\section{Communication Unit}

\section{Zigbee}

The Zigbee is the technology which is used to carry less data rates but it will transfer the data to very long distances. The Zigbee will receive the data from the microcontroller and will send to the control section. In the control section the Zigbee technology is used to send the commands from the user to the microcontroller. 


\section{Device Driver Unit \\ Motor Driving}

The motors of our robot will be controlled by using the driving circuit in which the ULN2003 driver IC will be used to provide the proper current rating to the motor. The sinking current of the ULN driver is around $500 \mathrm{ma}$.

\section{Display Unit}

A liquid crystal display (LCD) is a flat panel display, video display that uses the light modulating properties of liquid crystals (LCs). Lcds do not emit light directly. The main use of this is to view the operations going on in the robot.

\section{Robot Unit}

The pick and place robot is used to pick the object through the command given by the controller and place the object in the desired location.

\section{LDR}

A Light Dependent Resistor (photoconductor, or photocell) is a device which has a resistance which varies according to the amount of light falling on its surface.

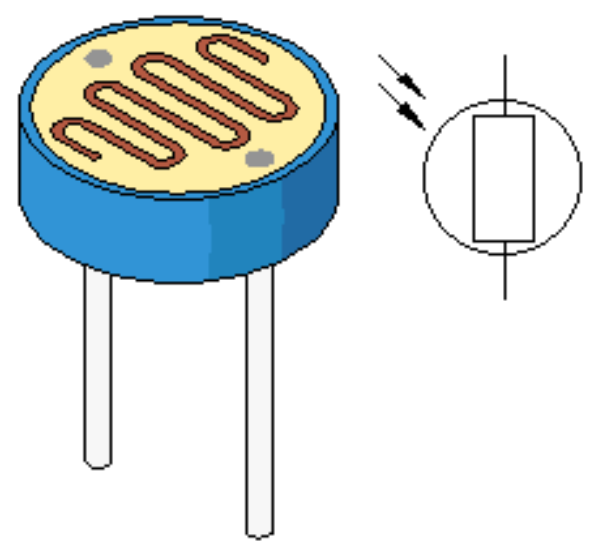

A typical light dependent resistor is pictured above together with circuit symbol. Different LDR's have different specifications, however some of the LDR's have fairly standard resistance of $1 \mathrm{MOhm}$ in darkness, and a resistance of a couple of kOhm in bright light (10-20kOhm @10 lux, 2-4kOhm @ 100 lux).Uses for Light Dependent Resistors

Light dependent resistors are a vital component in any electric circuit which is to be turned on and off automatically according to the level of ambient light - for example, solar powered lighting for security and gardening. An LDR can even be used in a simple remote control circuit using the backlight of a mobile phone to turn on a device - call the mobile from anywhere in the world, it lights up the LDR, and lighting (or a garden sprinkler) can be turned on remotely.

\section{Stepper Motor}

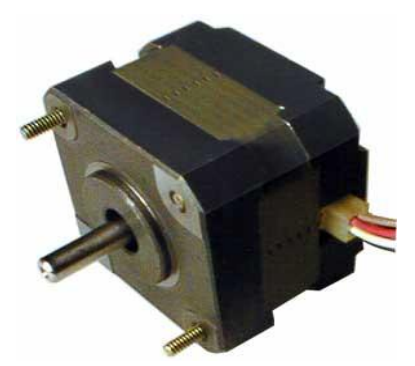

A stepper motor is a brushless, synchronous electric motor that converts digital pulses into mechanical shaft rotation. Every rotation of the stepper motor is divided into a discrete number of steps, and the motor must receive a separate pulse for each step. The size of each step is same and the stepper motor can only take one step at a time. Since each pulse causes the motor to rotate a precise angle, typically $1.8^{\circ}$, the motor's position can be 
controlled without any feedback mechanism. The speed of rotation directly proportional to the frequency of the pulses. Stepper motors are used every day in both industrial and commercial applications because of their low cost, high fidelity, high torque at low speeds and a simple, strenuous construction that operates in almost any environment.

\section{Conclusion}

The interest of this robotic system lies in the design concept, based on master and slave based microcontroller. On this basis, our proposal presents the construction of a solar tracking mechanism aimed at increasing the rover's power regardless of its mobility. On the other hand, it proposes zigbee based wireless communication to control and move the robot. The aim is completing the process of controlling the robot in the availability of solar power.

\section{Future Enhancement}

We are implementing a prototype of the robot in which it performs only some limited actions, if we want to implement in real time, with advanced technology and with latest designing we can achieve the robot perfectly in order to work in real time.

\section{References}

[1] N.Wilhelm, B.W. Surgenor, and J. G. Pharoah, "Design and Evaluation of amicro-fuel-cell-based power system for a mobile robot," IEEE/ASMETrans. Mechatronics, vol. 11, no. 4, pp. 471-476, Aug. $2006 .$.

[2] Anderson, I. A. Ieropoulos, T.McKay, B. O’Brien, and C. Melhuish, "Power for robotic artificial muscles," IEEE/ASME Trans. Mechatronics, vol. 16, no. 1, pp. 107-111, Feb. 2011.

[3] Jaw-KuenShiau, Member, IEEE, Der-Ming Ma, Pin-Ying Yang, Geng-Feng Wang, JhijHua Gong, "Design of a Solar Power Management System for an Experimental UAV", IEEE Transactions On Aerospace And Electronic Systems Vol. 45, No. 4 October 2009

[4] J. E. Gonz'alez Ramos, "Battery charging optimization with steerable solar cells," M.S. thesis, Dept. Electron. Eng., Comput. Syst. Autom., Universidad de Huelva, Huelva, Spain, 2010.

[5] David Appleyard, "Solar Trackers: Facing the Sun", Renewable Energy World Magazine, UK: Ralph Boon, June 1, 2009.

[6] B. Afarulrazi,W. M. Utomo, K. L. Liew, and M. Zafari, "Solar tracker robot using microcontroller," in Proc. Int. Conf. Bus., Eng. Ind. Appl., 2011, pp. 47-50.

[7] T. Jinayim, S. Arunrungrasmi, T. Tanitteerapan, and N. Mungkung, "Highly efficient low power consumption tracking solar cells for white-LED based lighting system,” World Acad. Sci., Eng. Technol., vol. 28, pp. 291-296, 2007.onf. Bus., Eng. Ind. Appl., 2011, pp. $47-50$.

[8] Adrian Catarius and Mario Christiner, "Azimuth-Altitude Dual Axis Solar Tracker”, A Master Qualifying Project: Submitted To The Faculty Of Worcester Polytechnic Institute In Fulfillment Of The Degree Of Bachelor Of Science, October 2009.

[9] Tom'as de J. Mateo Sanguino and Justo E. Gonz'alez Ramos, "Smart Host Microcontroller for Optimal Battery Charging in a SolarPowered Robotic Vehicle", IEEE/ASMETransactions On Mechatronics,April 2012. 